\title{
CLASSIFICAÇÃO DE SUPERFÍCIES IMPERMEÁVEIS EM IMAGEM MULTIESPECTRAL COM ALGORITMO DE MACHINE LEARNING
}

\section{Classification of Impervious Surfaces in Multispectral Image with Machine Learning Algorithm}

Michelle Tais Garcia Furuya, Danielle Elis Garcia Furuya, Lucas Prado Osco, Ana Paula Marques Ramos.

Universidade do Oeste Paulista - UNOESTE, Presidente Prudente, SP. E-mail: michellegfuruya@gmail.com

RESUMO - O processo de urbanização expõe a paisagem urbana a rápidas e constantes transformações. A mudança nos padrões de uso e cobertura do solo impacta diretamente na qualidade de vida das cidades. Por isso o monitoramento da composição territorial urbana se torna fundamental para a gestão urbana. Para obter acesso a esses dados, estudos vem aplicando técnicas de sensoriamento remoto aliada à aprendizagem de máquina (machine learning). As imagens de satélites oferecem dados em larga escala e com alta resolução temporal, facilitando a detecção de mudanças na paisagem. Já os algoritmos de machine learning proporcionam classificações com maior acurácia em relação aos métodos tradicionais. A partir desse contexto e das técnicas disponíveis, o estudo tem como objetivo avaliar o desempenho do algoritmo Support Vector Machine (SVM) em quantificar áreas impermeáveis no perímetro urbano de Presidente Prudente a partir de uma imagem Planet. O processo de classificação foi feito por meio do software ArcGIS Pro. Os resultados demonstram alto desempenho para o SVM quando aplicado em classificação de áreas impermeáveis em território urbano. A acurácia de $94 \%$ evidencia que o método proposto no trabalho é útil como ferramenta para o planejamento urbano.

Palavras-chave: classificação; inteligência artificial; superfícies impermeáveis.

ABSTRACT - The urbanization process exposes the urban landscape to rapid and constant transformations. The change in land use and land cover patterns directly impacts the quality of life in cities. Therefore, monitoring the urban territorial composition becomes essential for urban management. To gain access to these data, studies have been applying remote sensing techniques combined with machine learning. Satellite images provide large-scale data with high temporal resolution, making it easier to detect changes in the landscape. Machine learning algorithms, on the other hand, provide classifications with greater accuracy compared to traditional methods. From this context and the available techniques, the study aims to evaluate the performance of the Support Vector Machine (SVM) algorithm in quantifying impervious areas in the urban perimeter of Presidente Prudente from a Planet image. The classification process was done using ArcGIS Pro software. The results demonstrate high performance for the SVM when applied in classification of 
impervious areas in urban territory. The accuracy of $94 \%$ shows that the method proposed in the work is useful as a tool for urban planning.

Keywords: classification; artificial intelligence; impervious surfaces.

\section{INTRODUÇÃO}

A paisagem urbana é composta e influenciada por diversos elementos. A expansão urbana produz a mudança da cobertura natural da terra para as superfícies antropogênicas impermeáveis, que podem ser superfícies impermeáveis naturais como rochas e geleiras ou superfícies impermeáveis antropogênicas como asfalto e concreto (LIN et al., 2020).

Compreender o processo de expansão urbana, especialmente a mudança no uso/ cobertura do solo é necessário para o planejamento e gestão urbana (DOU e KUANG, 2020). Nesse contexto, as áreas impermeáveis são importantes na análise e gestão das cidades, pois estas áreas determinam um aumento da temperatura da superfície do solo e estresse hídrico das árvores por meio da absorção e armazenamento de energia solar durante o dia (MORABITO et al., 2021).

O monitoramento e análise de determinadas áreas podem ser feitos com auxílio do sensoriamento remoto, pois permite obter conhecimento relevante para processos sociais, ecológicos e inovações tecnológicas (WELLMANN et al., 2020). O mapeamento baseado em imagens de satélite fornece uma ferramenta essencial para avaliar os padrões de atividade de uso da terra atuais e históricos (RAHMAN et al., 2020). Além disso, as imagens de sensoriamento remoto, com alta resolução espacial, possuem um grande potencial para extrair e analisar funções da região urbana (XU et al., 2020).

Com base na literatura é possível observar estudos que analisaram superfícies impermeáveis com diferentes imagens de satélite. Kesikoglu et al., 2021 mostraram resultados promissores na identificação de áreas impermeáveis com a utilização do algoritmo Support Vector Machine (SVM) e imagens Landsat. Tang et al., (2020) obtiveram resultados satisfatórios com a utilização do algoritmo Random Forest (RF) para identificar áreas impermeáveis com imagens Sentinel-2.

As imagens Planet contêm 4 bandas, apresentam alta resolução espacial (com variação de 1 a 5 metros) e proporcionam análises eficientes (AGUILAR; KUFFER, 2020). Estudos realizados com imagens Planet obtiveram resultados satisfatórios para análise de alvos, inclusive em ambientes urbanos. Michael et al 2018, avaliaram a capacidade de detecção de queima em vegetação urbana com imagens Planet e três índices de vegetação. $O$ estudo obteve uma precisão geral de $87 \%$. McLaughlin et al 2019, também obtiveram resultados significantes com a utilização de imagens Planet para detectar a mudança no nível de vegetação.

A aprendizagem de máquina (Machine Learning - ML) corresponde a uma subárea da Inteligência Artificial. As técnicas de ML consistem na aplicação de um ou mais algoritmos para a realização de uma determinada tarefa e podem ser aplicados à diversas imagens de alta resolução (MISRA et al., 2020). Um dos algoritmos de ML mais utilizados é o Support Vector Machine (SVM). Segundo Misra et al 2020, a maioria dos pesquisadores recomendam o uso do SVM para extração de dados com alta resolução.

Estudos realizados em áreas urbanas obtiveram resultados significativos com a utilização de imagens de satélite e algoritmos de aprendizagem de máquina. Avudaiammal et al 2020, analisaram a capacidade do algoritmo SVM para detectar edificações em áreas urbanas com imagens Sentinel-2 e obtiveram acurácia acima de $90 \%$. Rahman et al 2020 , utilizaram algoritmos de destaque na área de aprendizagem de máquina como o RF e SVM para identificar a expansão em ambientes urbanos e rurais. Os autores utilizaram imagens Landsat, Sentinel e Planet e o estudo mostrou que o SVM obteve os melhores resultados com acurácia acima de $90 \%$.

No contexto de aprendizagem de máquina, o objetivo deste estudo foi analisar o desempenho do algoritmo SVM em identificar e quantificar áreas impermeáveis no perímetro urbano com imagem Planet.

\section{METODOLOGIA}

A área de estudo corresponde ao perímetro urbano de Presidente Prudente, município localizado no interior do estado de São 
Paulo. Com população superior a 200.000 habitantes (IBGE, 2021), é conhecida como a capital do Oeste Paulista por ser o polo de comércios e serviços da região. Por se tratar de uma cidade de destaque e em constante expansão, o mapeamento de superfícies impermeáveis torna-se fundamental para o planejamento urbano.

O estudo foi feito com base em uma imagem Planet de 22 de abril de 2021 conforme figura abaixo. A imagem apresenta resolução espacial de 3 metros e está projetada em Universal Transversa de Mercator (UTM), zona 22S - World Geodetic System (WGS) 84.

Figura 1. Imagem Planet do perímetro urbano de Presidente Prudente

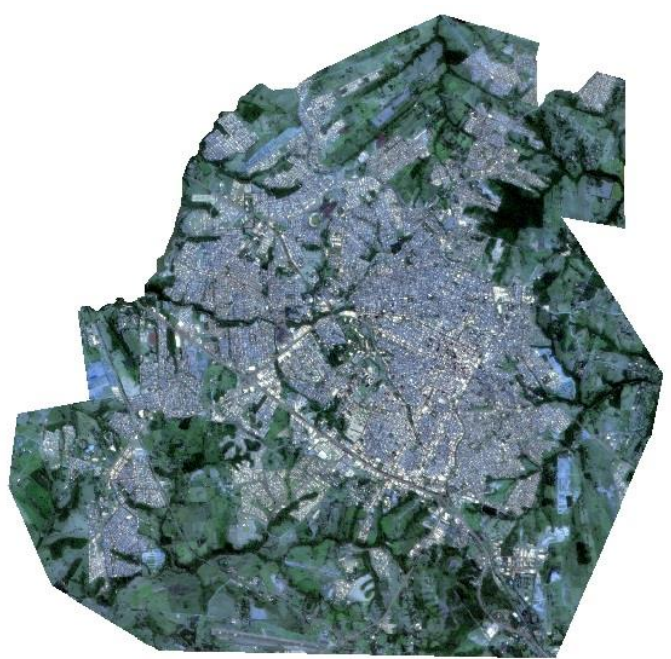

Fonte: Imagem Planet adaptada pelo autor (2021).

A imagem acima está em composição de cor natural. Entretanto é possível alterar a combinação de bandas, ou seja, atribuir uma composição de falsa cor, para destacar uma classe de interesse. Como a intenção é diferenciar áreas impermeáveis de áreas permeáveis, a composição colorida com a banda infravermelha é interessante por destacar a classe de vegetação em vermelho. Além disso as classes impermeáveis, como áreas construídas e rodovias, aparecem com alto contraste em relação ao vermelho, facilitando a distinção entre classes. $O$ processo de classificação, portanto, foi feito com base na composição de bandas 432 conforme figura abaixo.
Figura 2. Imagem Planet do perímetro urbano de Presidente Prudente em composição falsa-cor

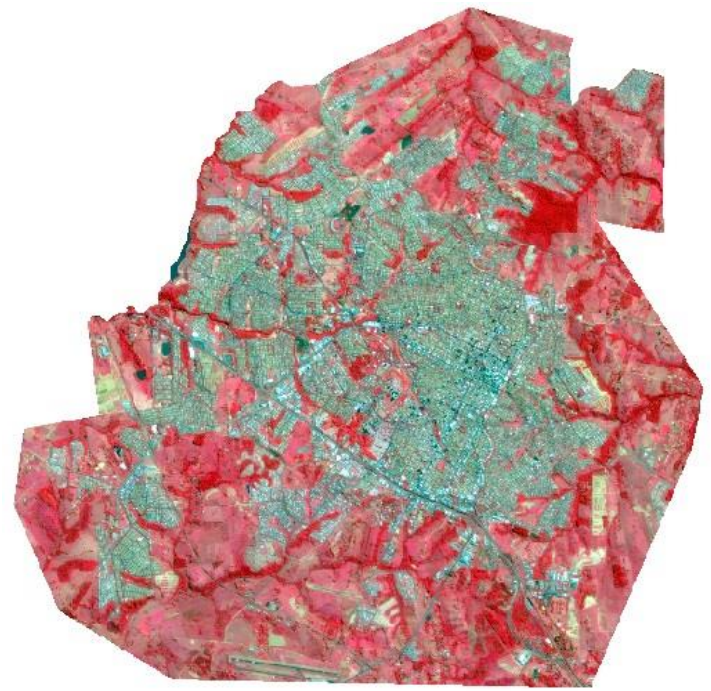

Fonte: Imagem Planet adaptada pelo autor (2021).

Para a realização do estudo foi feita a divisão dos procedimentos em etapas que compõem a metodologia. As etapas estão organizadas segundo fluxograma da figura $3 \mathrm{e}$ foram realizadas no software ArcGIS Pro, disponibilizado pela ESRI por meio de licença estudantil.

Figura 3. Fluxograma da metodologia

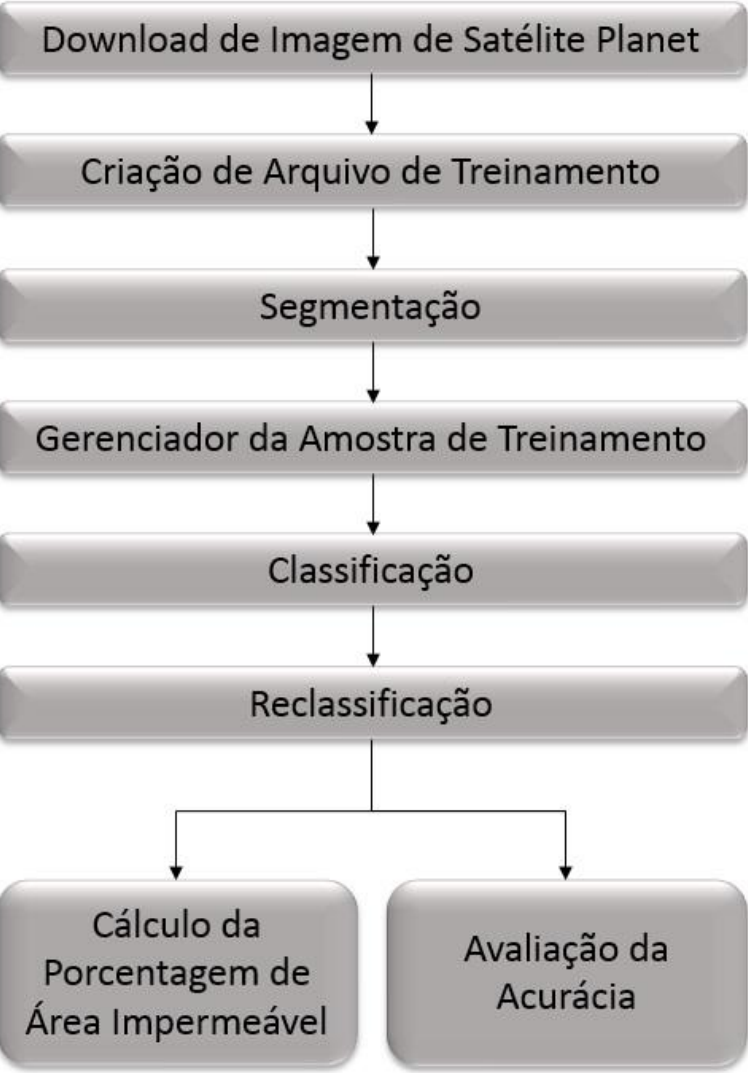

Fonte: Os autores. 


\subsection{Criação do Arquivo de Treinamento}

$O$ arquivo de treinamento é necessário por se tratar de uma classificação supervisionada, ou seja, antes de realizar a classificação é preciso ensinar o algoritmo a entender o que são áreas impermeáveis e áreas permeáveis (ESRI, 2021a). Para isso cria-se polígonos que representem cada classe desejada. Esses polígonos serão as amostras de treinamento utilizadas como base para o algoritmo.

No arquivo de treinamento desse estudo foram vetorizados 100 polígonos no total, sendo 50 polígonos referentes às áreas impermeáveis e 50 polígonos correspondendo às áreas permeáveis. A quantidade de polígonos é suficiente para que a distribuição seja feita por toda a imagem. A vetorização foi feita com base na imagem de satélite e é mostrada na figura 4. Os polígonos de área permeável estão representados pela cor verde enquanto os polígonos de área impermeável aparecem em vermelho.

Figura 4. Amostras de treinamento

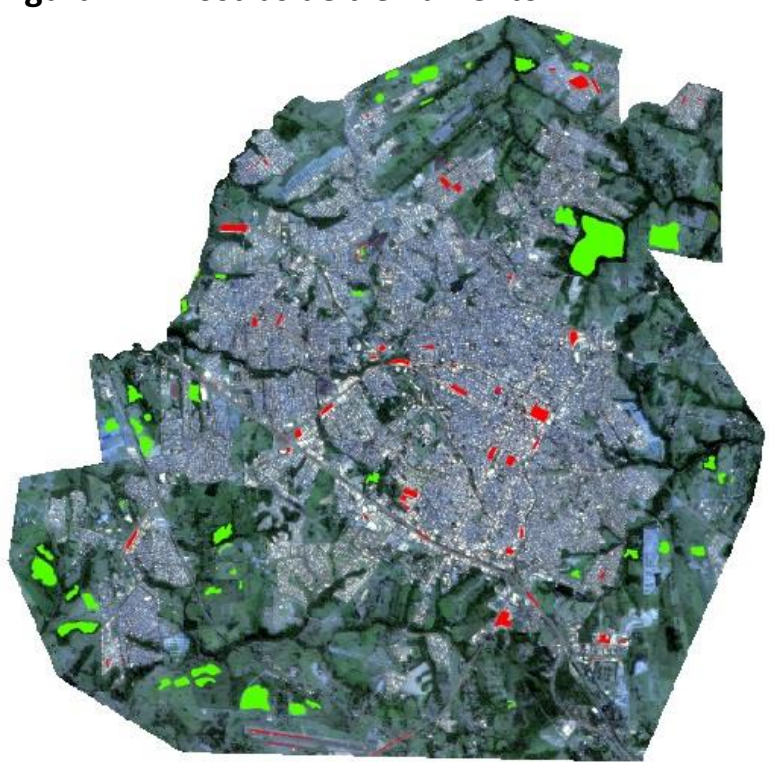

Fonte: Os autores.

Como o intuito do estudo é diferenciar somente duas classes (área impermeável e área permeável) os polígonos foram criados em diferentes superfícies para que o algoritmo compreenda que classes menores, nesse caso, se encaixam na mesma categoria. Por exemplo, os polígonos de área permeável incluem vegetação, pastagem, solo exposto e corpos d'água. Da mesma forma, áreas impermeáveis também incluem diferentes classes na mesma categoria como área construída, rodovias e ruas.
A figura 5a mostra um exemplo de polígonos de área permeável em vegetação e solo exposto. A figura $5 \mathrm{~b}$ mostra polígonos de área impermeável em coberturas de edificações. Por fim, a figura 5c mostra polígonos de área permeável em pastagens e solo exposto e polígonos de área impermeável na pista do aeroporto de Presidente Prudente, para exemplificar que diferentes superfícies foram consideradas na criação do arquivo de treino.

Figura 5. Exemplos de polígonos de áreas permeáveis e áreas impermeáveis
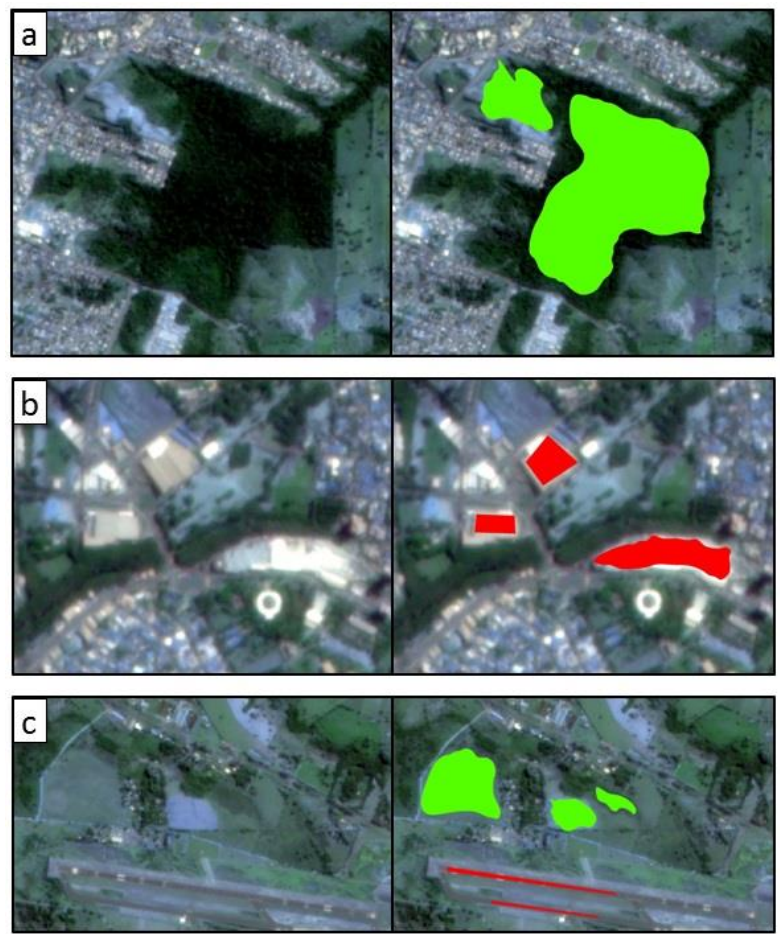

Fonte: Os autores.

\subsection{Segmentação}

A primeira etapa da classificação é a segmentação, cujo objetivo é agrupar pixels vizinhos que apresentam resposta espectral similar. Em outras palavras cada classe é identificada por uma mesma região e separada das demais classes. Essa etapa é necessária porque o processo da classificação será feito com base em objetos e não em pixels (ESRI, 2021a).

A segmentação é composta por três parâmetros: detalhe espectral, detalhe espacial e tamanho mínimo do segmento em pixels. Os valores do detalhe espectral e detalhe espacial variam de 1 a 20 . Valores de detalhe espectral altos são indicados quando deseja-se obter uma classificação mais detalhada, ou seja, quando componentes com características espectrais semelhantes precisam ser classificados em classes diferentes (ESRI, 2021a). Um exemplo de 
classificação detalhada com alto valor de detalhe espectral é a separação de diferentes espécies vegetais em classes distintas. Já um valor baixo inclui todas as espécies em uma única classe, denominada vegetação.

O detalhe espacial por sua vez, refere-se a pixels espacialmente próximos, mas espectralmente diferentes (ESRI, 2021a). Valores baixos podem ser aplicados em áreas impermeáveis onde não há necessidade de separar ruas e rodovias de áreas construídas por exemplo. Da mesma forma, valores altos são recomendados quando as categorias citadas precisam corresponder a classes diferentes.

Por fim o tamanho mínimo do segmento em pixels corresponde à unidade mínima de mapeamento do projeto. $O$ valor padrão indicado pelo software para este parâmetro é 20 (ESRI, 2021a).

O resultado da segmentação é mostrado na figura 6. Após testar diferentes valores, os parâmetros utilizados foram 15 para detalhe espectral, 15 para detalhe espacial e 20 para o tamanho mínimo do segmento em pixels. É interessante destacar que mesmo trabalhando com grandes classes, os valores espectrais e espaciais altos obtiveram melhores resultados em relação aos valores baixos. Isso porque no caso da imagem utilizada, os valores altos foram capazes de identificar construções isoladas. Por isso há a recomendação de testar diferentes valores para descobrir qual melhor se adapta a situação.

Figura 6. Segmentação da imagem Planet

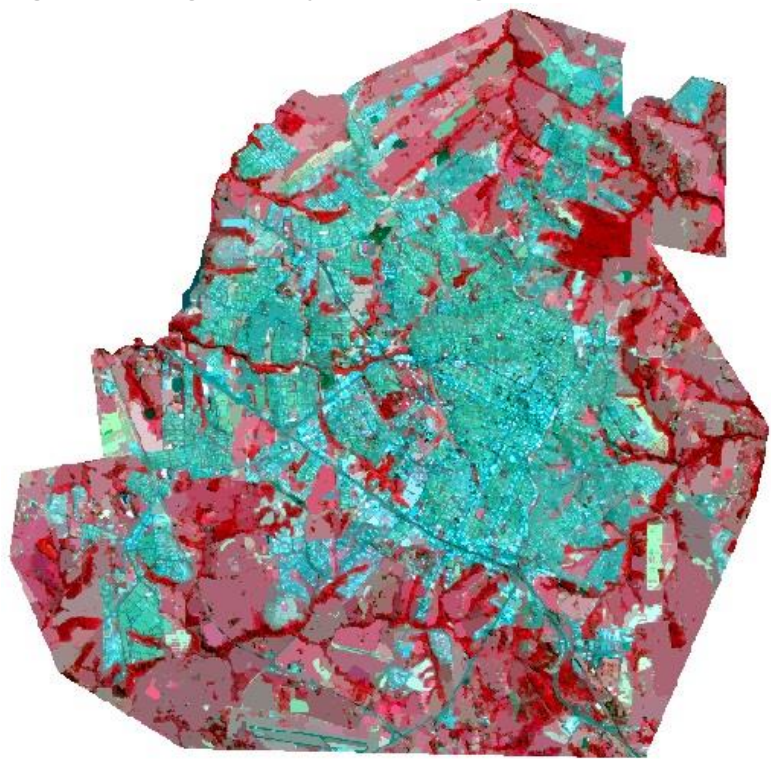

Fonte: Os autores.
Para melhor visualização, a figura 7 mostra um detalhe da imagem Planet comparando a composição em falsa cor e a imagem segmentada. É possível notar como o processo de segmentação agrupa pixels semelhantes em uma mesma região, facilitando, assim, a identificação de classes. O fragmento da imagem contém áreas de vegetação, área construída e parte do Balneário da Amizade.

Figura 7. (a) Detalhe da composição em falsacor. (b) Detalhe da segmentação

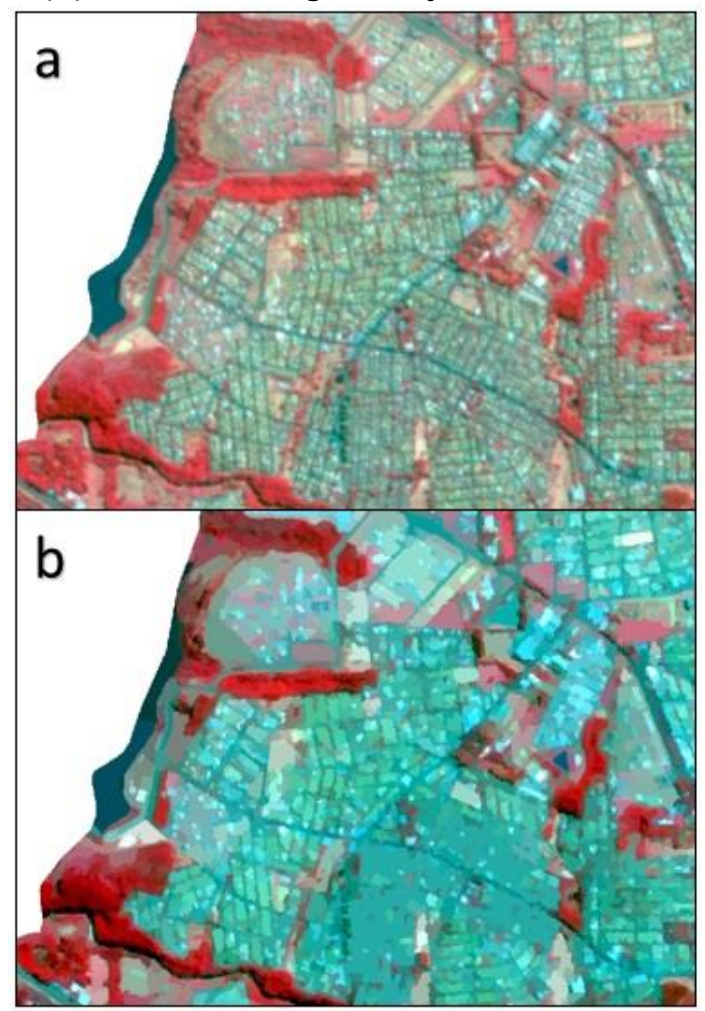

Fonte: Os autores

A imagem segmentada é a imagem usada pelo algoritmo de aprendizagem de máquina para realizar a classificação. Porém, conforme explicado na seção 2.1, para que a classificação possa ser feita, é necessário fornecer ao algoritmo amostras de treinamento que indiquem o que são áreas impermeáveis e permeáveis. Portanto, após a segmentação é necessário anexar o arquivo de treino por meio do Gerenciador da Amostra de Treinamento.

\subsection{Classificação}

Após inserir o arquivo de treinamento a classificação é iniciada por meio da ferramenta Assistente de Classificação. 0 método da classificação é supervisionado e baseado em objetos. 
Na etapa de treinamento do algoritmo há três opções de classificador: Maximum Likelihood (Máxima Verossimilhança), Random Trees (RT) e Support Vector Machine (SVM). Para o presente estudo o algoritmo selecionado foi o Support Vector Machine (SVM).

\section{RESULTADOS}

A classificação feita pelo algoritmo SVM é mostrada abaixo. As áreas permeáveis aparecem em marrom e as áreas impermeáveis são mostradas em cinza.

Figura 8. Classificação da imagem Planet

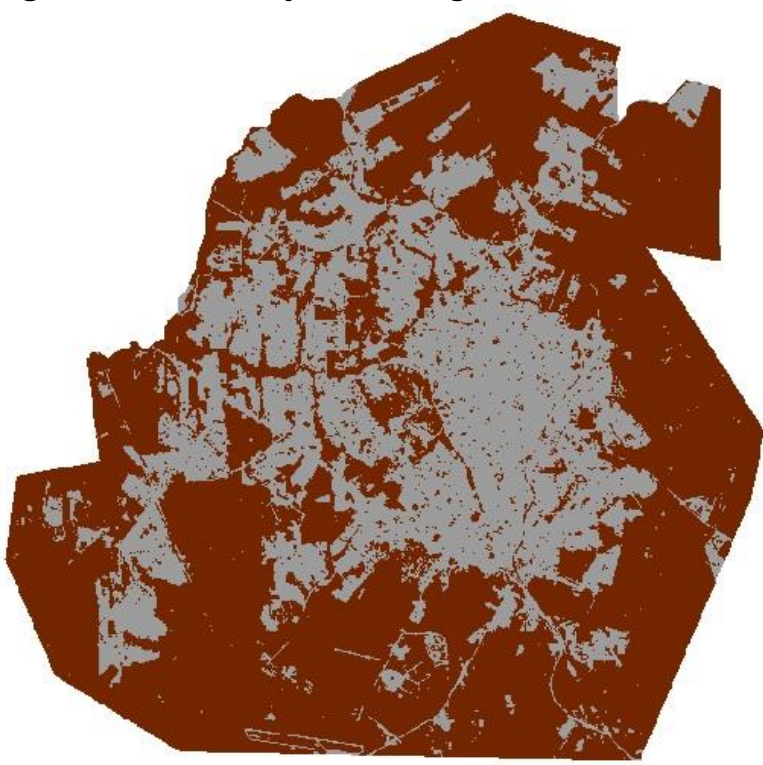

Fonte: Os autores.

Com base no resultado obtido é fundamental verificar se o algoritmo classificou as áreas corretamente. Tal verificação pode ser feita com base na imagem original.

Um exemplo da comparação realizada é mostrado na figura 9. Na imagem é possível perceber que parte do Balneário da Amizade foi classificado como área impermeável quando deveria ter sido classificado como área permeável, por se tratar de um corpo d'água.
Figura 9. Detalhe da classificação

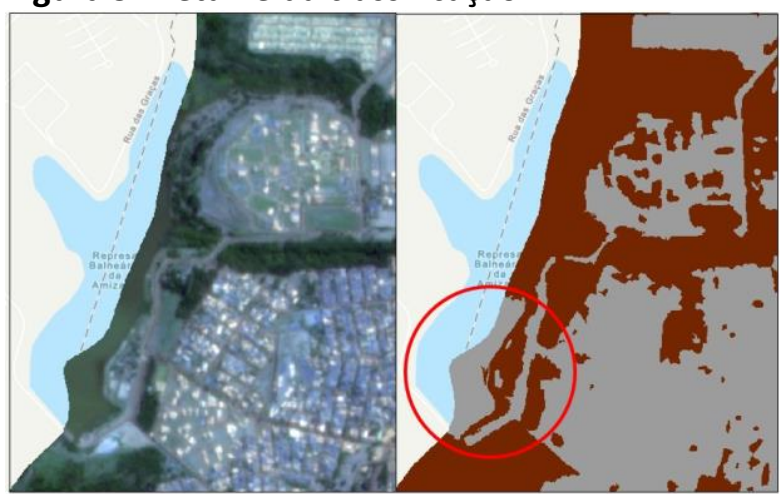

Fonte: Os autores.

Para minimizar os erros da classificação é possível aplicar um pós-processamento de modo que as áreas possam ser classificadas na categoria correta. Essa etapa é denominada Reclassificação e é recomendada para que não seja necessário realizar o procedimento do início novamente (ESRI, 2021a).

A reclassificação é mostrada na figura 10 . As áreas permeáveis aparecem em azul e as áreas impermeáveis são mostradas em cinza.

Figura 10. Reclassificação da Imagem Planet

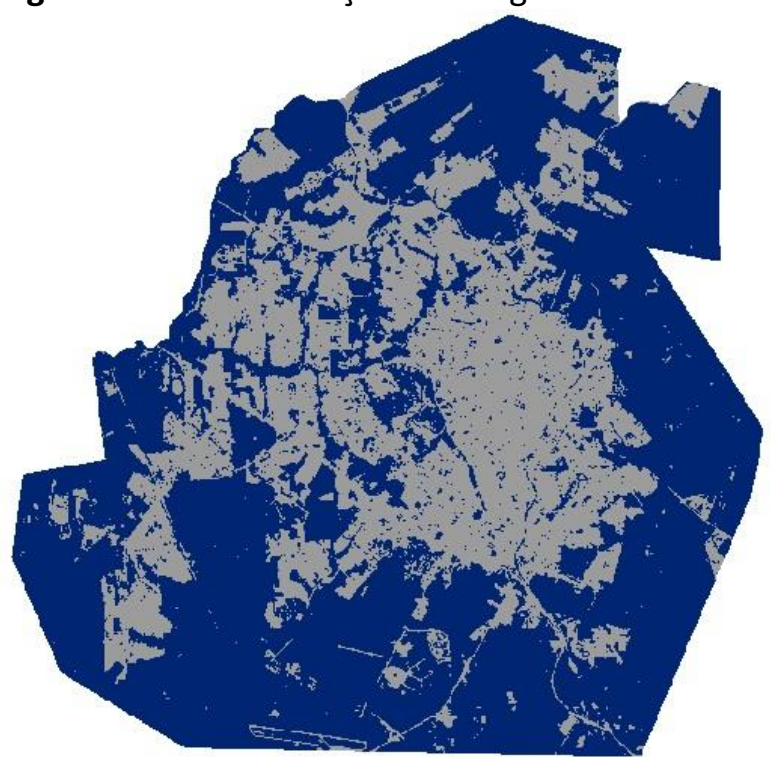

Fonte: Os autores.

Com a reclassificação, o detalhe do Balneário da Amizade mostrado anteriormente, além de outros erros identificados na etapa de classificação, foram corrigidos. A imagem abaixo mostra o detalhe do Balneário da Amizade. 
Figura 11. Detalhe da reclassificação

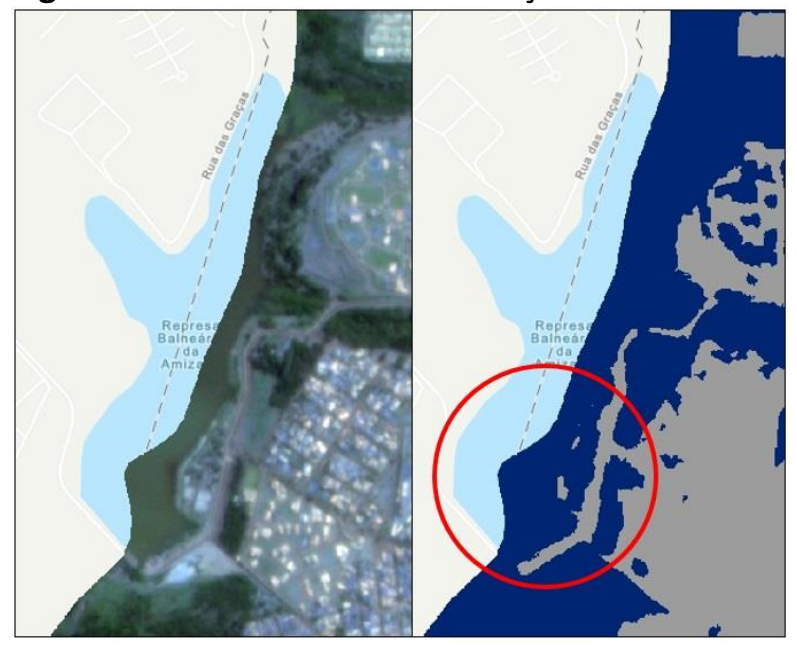

Fonte: Os autores.

3.1. Cálculo da Porcentagem de Área Impermeável

O cálculo da porcentagem de cada classe é feito com base na área. $O$ arquivo está no sistema UTM, portanto a área de cada classe que consta na tabela de atributos está em metros quadrados. Como o estudo engloba o perímetro urbano de uma cidade de médio porte, trabalhar com a área em quilômetros quadrados se torna mais interessante. Portanto após a conversão da unidade de medida da área, o cálculo da porcentagem foi feito pela razão entre a área de cada classe e a área total multiplicada por cem. 0 quadro 1 mostra o resultado obtido para cada classe.

Quadro 1. Porcentagem de cada classe

\begin{tabular}{|c|c|c|}
\hline CLASSE & ÁREA EM KM ${ }^{2}$ & PORCENTAGEM \\
\hline PERMEÁVEL & 78,92 & $64,19 \%$ \\
\hline IMPERMEÁVEL & 44,04 & $35,81 \%$ \\
\hline
\end{tabular}

Fonte: Os autores.

O resultado mostra que $64,19 \%$ do perímetro urbano de Presidente Prudente corresponde a áreas permeáveis o que inclui vegetação, corpos d'água e solo exposto. Já as edificações, ruas e rodovias que integram a classe de área impermeável ocupam $35,81 \%$ do perímetro urbano.

\subsection{Avaliação da Acurácia}

A avaliação do resultado da reclassificação é feita por meio da análise da matriz de confusão. A matriz apresenta informações sobre as classificações reais e previstas, evidenciando a frequência com que determinado dado é classificado na classe correta (DENG et al. 2016; RUUSKA et al. 2018). A matriz de confusão gera diferentes métricas para avaliar a acurácia, ou seja, o desempenho ou nível de acerto do algoritmo.

Para construção da matriz de confusão é necessário criar um arquivo de pontos (ESRI, 2021b). A quantidade de pontos é especificada de acordo com a área geográfica. Para este estudo verificou-se que 100 pontos é suficiente. Além disso a estratégia de amostragem escolhida é do tipo Estratificado Aleatório o que indica que além dos pontos serem gerados aleatoriamente sobre a imagem, a quantidade de pontos será maior na classe predominante, ou seja, na classe de área permeável conforme mostrado no item 3.1. A figura 12 mostra a localização dos pontos na imagem.

Figura 12. Arquivo de pontos gerado para a construção da matriz de confusão

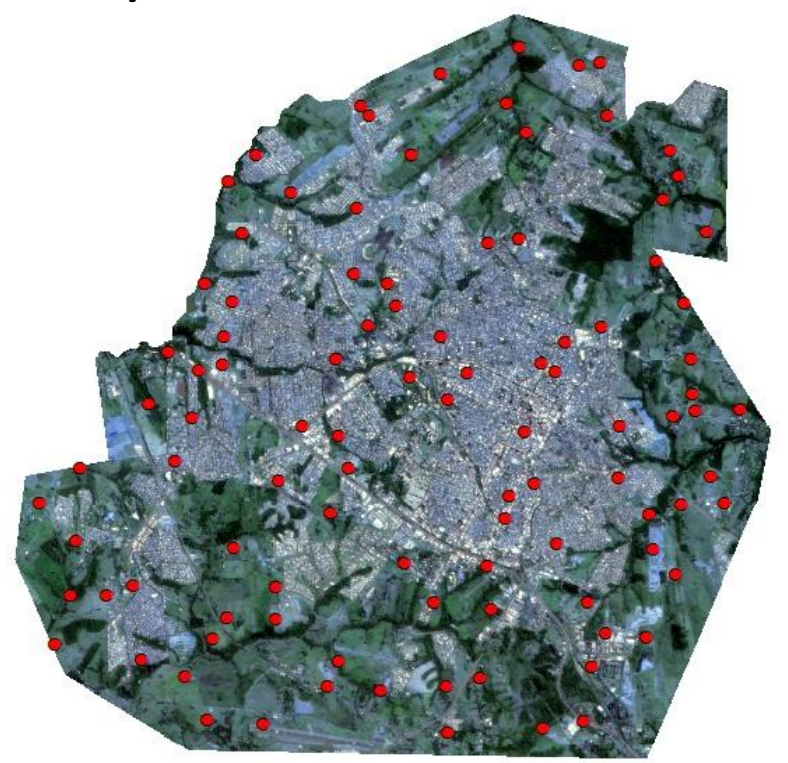

Fonte: Os autores.

$\mathrm{Na}$ tabela de atributos do arquivo de pontos há uma coluna que indica a classe atribuída para cada ponto e outra coluna com o ground truth ou dados de refêrencia, que indica a classe verdadeira do ponto. A coluna do groud truth é preenchida manualmente por meio da verificação ponto a ponto com base na imagem Planet original. Em outras palavras as duas colunas são uma comparação entre a classe atribuída pelo software e a classe correspondente na vida real. As duas colunas são relacionadas pela matriz de confusão posteriormente. 
A figura 13 mostra a matriz de confusão referente ao processo de reclassificação feita pelo algoritmo SVM.

Figura 13. Matriz de confusão da reclassificação

\begin{tabular}{|c|c|c|c|}
\hline & \multicolumn{2}{|c|}{ VALOR REAL. } \\
\hline & & IMPERMEÁVEL & PERMEÁVEL \\
\hline \multirow{2}{*}{ 응 } & IMPERMEÁVEL & 31 & 5 \\
\hline & PERMEÁVEL & 1 & 63 \\
\hline
\end{tabular}

Fonte: Os autores.

Dos 100 pontos gerados na imagem, 64 correspondem à área permeável (por ser a classe predominante) e 36 foram gerados na classe impermeável. De acordo com a matriz de confusão é possível verificar que 31 pontos, do total de 36 , foram classificados como verdadeiro positivo, ou seja, o algoritmo SVM classificou como área impermeável quando o dado também correspondia à área impermeável na vida real. Já o verdadeiro negativo apresenta 63 pontos em um total de 64, o que indica que o algoritmo obteve somente um erro para classificar áreas permeáveis. 5 pontos foram classificados como falso positivo (classificado como impermeável quando deveria ser permeável) e somente 1 ponto corresponde ao falso negativo (classificado como permeável quando deveria ser impermeável). Com base nesses resultados 0 algoritmo alcançou acurácia de $94 \%$, o que indica um excelente desempenho.

\section{DISCUSSÃO}

Técnicas de sensoriamento remoto têm sido amplamente aplicadas em estudos urbanos visto que as imagens de satélite abrangem extensas áreas, possibilitando trabalhos em diferentes escalas, além de alta resolução temporal, disponibilizando dados com maior frequência. Como complementação, o uso de algoritmos de aprendizagem de máquina vem se tornando cada vez mais presente para estudos urbanos, incluindo de superfícies impermeáveis. Nesse sentido, o algoritmo SVM aparece como alternativa para a classificação de imagens multiespectrais, pois vem alcançando alta acurácia e apresentando resultados satisfatórios.

Zhang et al. (2018), realizaram o mapeamento de superfície impermeável na aglomeração urbana de Wuhan (WUA) - China em uma escala de tempo sazonal utilizando informações temporais de 2000 a 2016. Os dados foram extraídos de imagens Landsat e a classificação foi feita com o algoritmo SVM. O estudo mostrou um crescimento anual de $20,10 \%$ de superfícies impermeáveis na área geográfica estudada, ao longo dos 16 anos. Além disso, o método proposto mostrou que o SVM alcançou alta acurácia e melhor desempenho quando comparado aos métodos tradicionais.

Da mesma forma, Zeng et al. (2019) avaliaram os padrões de cobertura do solo urbano, incluindo superfícies impermeáveis, na cidade de Shenzhen - China. O estudo utiliza imagens Landsat 8 entre o período de 2013 a 2017 e realiza três abordagens para avaliar o desempenho da classificação: SVM, Máxima Verossimilhança e Rede Neural Artificial. O estudo conclui que a aplicação do algoritmo SVM atinge uma classificação de alta precisão, com acurácia superior a $90 \%$.

\section{CONSIDERAÇÕES FINAIS}

O estudo apontou alto desempenho do algoritmo Support Vector Machine (SVM) quando aplicado em classificação de área impermeável em território urbano. Com base em uma única imagem Planet e um arquivo de treino, o algoritmo foi capaz de agrupar diferentes feições em apenas duas classes (áreas impermeáveis e permeáveis) alcançando acurácia de $94 \%$ após a etapa de reclassificação. Com os mesmos dados também foi possível calcular a porcentagem de cada classe. Portanto com os resultados obtidos é possível afirmar que a aplicação de algoritmos de machine learning em processos de classificação de imagens Planet se torna uma ferramenta útil para o planejamento urbano.

\section{AGRADECIMENTOS}

Os autores agradecem o apoio financeiro da Coordenação de Aperfeiçoamento de Pessoal de Nível Superior - CAPES.

\section{REFERÊNCIAS}

AGUILAR, R.; KUFFER, M. Cloud computation using high-resolution images for improving the SDG indicator on open spaces. Remote Sens., 12, 1144, 2020 https://doi.org/10.3390/rs12071144

AVUDAIAMMAL, R.; ELAVENI, P.; SELVAN, S.; RAJANGAM, V.Extraction of Buildings in Urban Area for Surface Area Assessment from Satellite Imagery based on Morphological Building Index using SVM Classifier. J. Indian Soc. Remote. 
Sens., v. 48, p. 1325-1344, 2020 https://doi.org/10.1007/s12524-020-01161-0

DENG, X.; LIU, Q.; DENG, Y.; MAHADEVAN, S. (2016). An improved method to construct basic probability assignment based on the confusion matrix for classification problem. Information Sciences, v. 340-341, p. 250-261. 2016. https://doi.org/10.1016/j.ins.2016.01.033

DOU, Y.; KUANG, W. A comparative analysis of urban impervious surface and green space and their dynamics among 318 different size cities in China in the past 25 years. Sci. Total Environ., v. 706, p. 135828.2020 https://doi.org/10.1016/i.scitotenv.2019.135828

ESRI - ARCGIS PRO The Image Classification Wizard. (2021a). Disponível em: https://pro.arcgis.com/en/pro-

app/latest/help/analysis/image-analyst/the-

image-classification-wizard.htm. Acesso em: 29 maio 2021.

ESRI - ARCGIS PRO. Accuracy Assessment. Disponível em: https://pro.arcgis.com/en/proapp/latest/help/analysis/image-analyst/accuracyassessment.htm. Acesso em: 08 jun. 2021.

IBGE - INSTITUTO BRASILEIRO DE GEOGRAFIA E ESTATÍSTICA. Cidades e Estados - Presidente Prudente. (2021). Disponível em: https://www.ibge.gov.br/cidades-eestados/sp/presidente-prudente.html. Acesso em: 03 ago. 2021.

KESIKOGLU, M.H.; OZKAN, C.;KAYNAK, T. The impact of impervious surface, vegetation, and soil areas on land surface temperatures in a semi-arid region using Landsat satellite images enriched with Ndaisi method data. Environ Monit Assess, v. 193, n. 143, 2021.. https://doi.org/10.1007/s10661-021-08916-3

LIN, Y.; ZHANG, H.; LIN, H.; GAMBA, P.; LIU, X. Incorporating synthetic aperture radar and optical images to investigate the annual dynamics of anthropogenic impervious surface at large scale. Remote Sens. Environ., v. 242, p. 111757, 2020.https://doi.org/10.1016/i.rse.2020.111757

MCLAUGHLIN, C.; HUTSON, H.; DE VINE, L.; WOODLEY, A.; GEVA, S.; CHAPPELL T. Change detection over the state of Queensland using high resolution Planet satellite mosaics, Digital Image Computing: Techniques and Applications (DICTA), $2019.2 \quad$ pp. 1-8. https://doi.org/10.1109/DICTA47822.2019.89459 $\underline{42}$

MICHAEL, Y.; LENSKY, I.M.; BRENNER, S.; TCHETCHIK, A.; TESSLER, N.; HELMAN, D. Economic Assessment of Fire Damage to Urban Forest in the Wildland-Urban Interface Using Planet Satellites Constellation Images. Remote $\begin{array}{llll}\text { Sens., } & 10, & 1479 . & 2018\end{array}$ https://doi.org/10.3390/rs10091479

MISRA, M., D. KUMAR, AND S. SHEKHAR. Assessing Machine Learning Based Supervised Classifiers for Built-Up Impervious Surface Area Extraction from Sentinel-2 Images. Urban Forestry \& Urban Greening, v. 53, p. 126714, 2020.

https://doi.org/10.1016/i.ufug.2020.126714

MORABITO, M.; CRISCI, A.; GUERRI, G.; MESSERI, A.; CONGEDO, L.; MUNAFÒ, M. Surface urban heat islands in Italian metropolitan cities: Tree cover and impervious surface influences. Sci. Total Environ., v. 751, p. 142334, 2021 https://doi.org/10.1016/j.scitotenv.2020.142334

RAHMAN, A; ABDULLAH, H.M; TANZIR, M.T; HOSSAIN, M.J; KHAN, B.M; MIAH, M.G; ISLAM, I. Performance of different machine learning algorithms on satellite image classification in rural and urban setup. Rem. Sens. Appl. Soc. Environ., v. 20, p. 10041, 2020. https://doi.org/10.1016/j.rsase.2020.100410

RUUSKA, S., HÄMÄLÄINEN, W., KAJAVA, S., MUGHAL, M., MATILAINEN, P., MONONEN, J. Evaluation of the confusion matrix method in the validation of an automated system for measuring feeding behaviour of cattle. Behavioural Processes, v. 148, p. 56-62, 2018 https://doi.org/10.1016/j.beproc.2018.01.004

TANG, P; DU, P; LIN, C; GUO, S; QIE. L. A novel sample selection method for impervious surface area mapping using JL1-3B nighttime light and Sentinel-2 imagery. IEEE J. Sel. Top. Appl. Earth Obs. Remote Sens. p. 1.2020. https://doi.org/10.1109/JSTARS.2020.3004654

WELLMANN, T.; LAUSCH, A.; ANDERSSON, E.; KNAPP, S.; CORTINOVIS, C.; JACHE, J.; SCHEUER, 
S.; KREMER, P.; MASCARENHAS, A.; KRAEMER, R.; et al. Remote sensing in urban planning: Contributions towards ecologically sound policies? Landsc. Urban Plan. 204, 103921, 2020 https://doi.org/10.1016/i.landurbplan.2020.1039 $\underline{21}$

$X U$, S.; QING, L.; HAN, L.; LIU, M.; PENG, Y.; SHEN, L. A New Remote Sensing Images and Point-ofInterest Fused (RPF) Model for Sensing Urban Functional Regions. Remote Sens. v. 12, p. 1032, 2020 https://doi.org/10.3390/rs12061032

ZENG, Q.; XIE, Y.; LIU, K. . Assessment of the patterns of urban land covers and impervious surface areas: A case study of Shenzhen, China. Physics and Chemistry of the Earth, v. 110, p. 17, 2019 https://doi.org/10.1016/i.pce.2019.04.002

ZHANG, L.; ZHANG, M.; YAO, Y. Mapping seasonal impervious surface dynamics in Wuhan urban agglomeration, China from 2000 to 2016. International Journal of Applied Earth Observation and Geoinformation, v. 70, p. 5161, 2018

https://doi.org/10.1016/j.jag.2018.04.005 